\title{
Simultaneous Surgical Treatment of Congenital Spinal Deformity Associated with Intraspinal Anomalies
}

\author{
Manoj Singrakhia ${ }^{1}$, Nikhil Malewar ${ }^{1}$, Sonal Deshmukh ${ }^{2}$, Shivaji Deshmukh ${ }^{2}$ \\ ${ }^{1}$ Department of Spine Surgery, Shanta Spine Institute, Nagpur, India \\ ${ }^{2}$ Department of Anaesthesia, Shanta Spine Institute, Nagpur, India
}

\section{Study Design: Prospective case series.}

Purpose: To study the safety, efficacy, and long-term outcomes of single-stage surgical intervention for congenital spinal deformity and intraspinal anomalies.

Overview of literature: Congenital spinal deformities associated with intraspinal anomalies are usually treated sequentially, first by treating the intraspinal anomalies followed by deformity correction after a period of 3-6 months. Recently, a single-stage approach has been reported to show better postoperative results and reduced complication rates.

Methods: Thirty patients (23 females and seven males) were prospectively evaluated for the simultaneous surgical treatment of congenital spinal deformity with concurrent intraspinal anomalies from May 2006 to October 2016. The average age at presentation was $9.8 \pm 3.7$ years, with the average follow-up duration being $49.06 \pm 8.6$ months. Clinical records were evaluated for clinical, radiological, perioperative, and postoperative data.

Results: The average angle of deformity was $56.53^{\circ} \pm 25.22^{\circ}$ preoperatively, $21.13^{\circ} \pm 14.34^{\circ}$ postoperatively, and $23.93^{\circ} \pm 14.99^{\circ}$ at the final follow-up. The average surgical time was $232.58 \pm 53.56$ minutes (range, 100-330 minutes), with a mean blood loss of $1,587.09 \pm 439.09 \mathrm{~mL}$ (range, $100-2,300 \mathrm{~mL}$ ).

Conclusions: Single stage surgical intervention for intraspinal anomalies with congenital spinal deformity correction, including adequate intra-operative wake-up test, is a viable option in appropriately selected patients and has minimum complication rates.

Keywords: Intraspinal anomaly; Congenital spinal deformity; Surgical treatment; Single stage

\section{Introduction}

The incidence of congenital scoliosis is 1-3 per 1,000 live births $[1,2]$ and that of intraspinal anomalies associated with congenital spinal deformity varies from $15 \%$ to $38 \%$ [3]. Congenital spinal deformity occurs during the first 8 weeks of gestation, during which the bony elements of the spine form and the neural axis completes its in-folding, closing the neural tube [4]. All these events simultaneously occur during fetal development, and any adverse event during this process can lead to congenital spinal deformities, such as scoliosis and/or kyphosis, along with

Received Aug 31, 2017; Revised Sep 23, 2017; Accepted Oct 2, 2017

Corresponding author: Manoj Singrakhia

Department of Spine Surgery, Shanta Spine Institute, 1st Floor Ashirvad Complex, Ramdaspeth, Nagpur 440010, Maharashtra, India

Tel: +91-09823080013, E-mail: singrakhia@hotmail.com 
intraspinal anomalies, including tethered cord, ArnoldChiari malformation, diastematomyelia, lipoma and lipomeningocele, teratoma, and syringomyelia $[2,5,6]$.

Intraspinal anomalies associated with spinal deformity can lead to rapid curve progression, progressive neurological deficit, and cardio-respiratory morbidity $[7,8]$. Such concurrent disorders are also associated with an increased risk of neurological deficit during surgery $[9,10]$. Congenital spinal deformities are often associated with intraspinal, genitourinary, cardiovascular, and other developmental anomalies [1].

The usual approach to the treatment of congenital spinal deformities associated with intraspinal anomalies is to first perform surgery for the intraspinal anomalies followed by deformity correction surgery 3-6 months later $[2,4,11]$. Staged surgery leads to scar formation, which distorts the normal anatomy and causes difficulties during the second surgical procedure, thereby increasing the chances of complications and possibly delaying the wound healing. Because both deformity correction and the treatment of intraspinal anomalies require same surgical approach, single-stage surgery would help prevent the complications associated with multi-stage procedures [7].

In the present study, we prospectively evaluated the safety and efficacy of the single-stage surgical correction of congenital spinal deformity associated with intraspinal anomalies in terms of perioperative and postoperative complications in 30 patients, with an average follow-up duration of $49.06 \pm 8.6$ months.

\section{Materials and Methods}

\section{Patients}

A total of 30 patients (23 females and seven males) with congenital spinal deformity (kyphosis and/or scoliosis) associated with intraspinal anomalies were prospectively evaluated from May 2006 to October 2016. Patients who presented with congenital spinal deformity associated with intraspinal anomalies (tethered cord, diastematomyelia, Arnold-Chiari malformation, or syringomyelia) were included, and those who presented with congenital spinal deformity secondary to other neuromuscular causes, including cerebral palsy, spinal muscular atrophy, or poliomyelitis, were excluded. All patients underwent simultaneous surgical intervention for intraspinal anomalies and congenital spinal deformity by a single surgeon at
Shanta Spine Hospital. Intra-operative wake-up test was conducted to assess any neurological deficit. Wake-up test consists of waking up the patient from anaesthesia during and after the completion of spinal procedure maintaining the position of the patient and the patient is asked to move their feet before continuation of anaesthesia. Two wake-up tests were conducted, with the first after the treatment of intraspinal anomaly and the second after deformity correction. Age, sex, associated anomalies, cutaneous markers, clinical presentation, neurological status, radiological features, intra-operative details, postoperative results, and complications were evaluated in all patients.

Standing anteroposterior and lateral views of the spine from C7 to sacrum were obtained to assess the deformity. The angle of deformity was calculated as per Cobb's method. A preoperative magnetic resonance imaging (MRI) of the area of interest along with the screening of the entire spine was performed to evaluate the associated intraspinal anomaly. The American Spinal Injury Association (ASIA) impairment scale was used to assess and monitor the neurological status preoperatively, postoperatively, and at each follow-up. All patients also underwent ultrasonography of the abdomen and echocardiography with a pediatrician's assessment to exclude any abdominal, genitourinary, or cardiac anomaly.

A written informed consent was taken from all the patients at Shanta Spine Institute included in the study.

\section{Surgical procedure}

All surgeries were performed using a posterior approach in the prone position. All patients presenting with congenital spinal deformity of $>40^{\circ}$ associated with an intraspinal anomaly underwent the single-stage treatment of intraspinal anomaly and deformity correction, and those presenting with hemivertebra with associated spinal cord deformity underwent the single-stage treatment of spinal anomaly and deformity correction even if the deformity was $<40^{\circ}$. During the surgery, the intraspinal anomaly was treated first (release of the tethered cord, excision of the diastematomyelic bone spurs, or decompression of Arnold-Chiari malformation or syringomyelia), followed by a wake-up test. After this, deformity correction was performed, again followed by a wake-up test. All patients underwent autologous local, iliac crest, or rib bone graft to achieve fusion. 

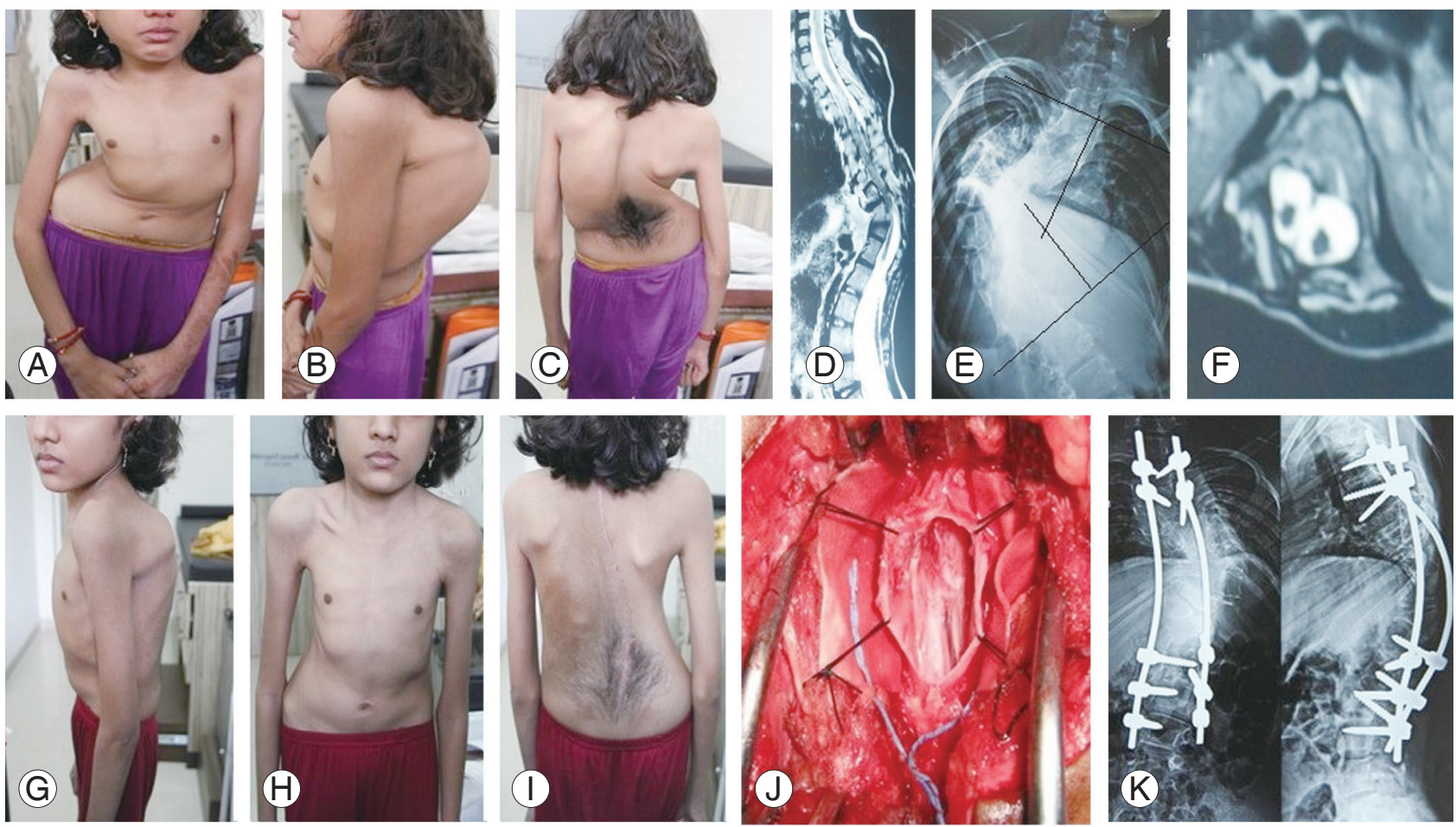

Fig. 1. A 9-year-old girl presented with deformity of the back with a hairy patch over the lumbar spine with diastematomyelia at D7-D8. She underwent the simultaneous resection of the bony spur at D7-8 and posterior instrumentation with pedicle screws and rod from D4 to L4. (A-C) Preoperative anterior, lateral, and posterior views of the patient showing severe kyphoscoliosis with hairy patch. (D) Preoperative MRI with sagittal view. (E) Preoperative $X$-ray showing severe kyphoscoliosis with an angle of $63^{\circ}$, with coronal imbalance of $0.5 \mathrm{~mm}$ and sagittal imbalance of $0.9 \mathrm{~mm}$. (F) Preoperative axial MRI showing diastematomyelia. (G-I) Postoperative images of the patient showing good correction and improved trunk balance. (J) Intra-operative images showing bony spur causing diastematomyelia. (K) Postoperative X-rays showing deformity correction with postoperative Cobbs angle of $23^{\circ}$, with coronal imbalance of $0.2 \mathrm{~mm}$ and sagittal imbalance of $0.1 \mathrm{~mm}$. MRI, magnetic resonance imaging.

\section{Results}

A total of 30 patients (23 females and seven males) who presented at our institute with congenital spinal deformity associated with intraspinal anomalies were included in the study. The mean age at presentation was $9.8 \pm 3.7$ years (range, 5-22 years). Of the 30 patients, 27 were children aged $<13$ years and three were adults aged $>13$ years. All patients underwent simultaneous spinal deformity correction and neurosurgical procedures. In our case series, 28 patients presented with congenital scoliosis (kyphoscoliosis was present in three patients among them) and two patients presented with kyphosis. Preoperative MRI revealed tethered cord in 13 patients (43.3\%), diastematomyelia in seven (23.3\%), Arnold-Chiari malformation in six (20.0\%), and syringomyelia in four (13.3\%).

The mean surgery time was $232.58 \pm 53.56$ minutes (range, 100-330 minutes), and the mean blood loss was $1,587.09 \pm 439.02 \mathrm{~mL}$ (range, $100-2,300 \mathrm{~mL}$ ). The average follow-up duration was $49.06 \pm 8.6$ months (Figs. 1, 2).

\section{Deformity correction}

The average angle of deformity was $56.52^{\circ} \pm 25.22^{\circ}$ preoperatively, $21.13^{\circ} \pm 14.34^{\circ}$ postoperatively, and $23.93^{\circ} \pm 14.99^{\circ}$ at the final follow-up. The mean correction rate was $62.46 \%$ in the immediate postoperative period. At the final follow-up, the mean loss of correction was $8.62 \%$ (Tables 1, 2).

\section{Complications}

Three patients in our study intraoperatively developed neurological deficits while deformity correction, identified during the wake-up test (one patient went from ASIA grade $\mathrm{C}$ to grade $\mathrm{B}$ and two went from ASIA grade $\mathrm{D}$ to grade B). All three patients underwent an immediate release of the correction, following which two patients 

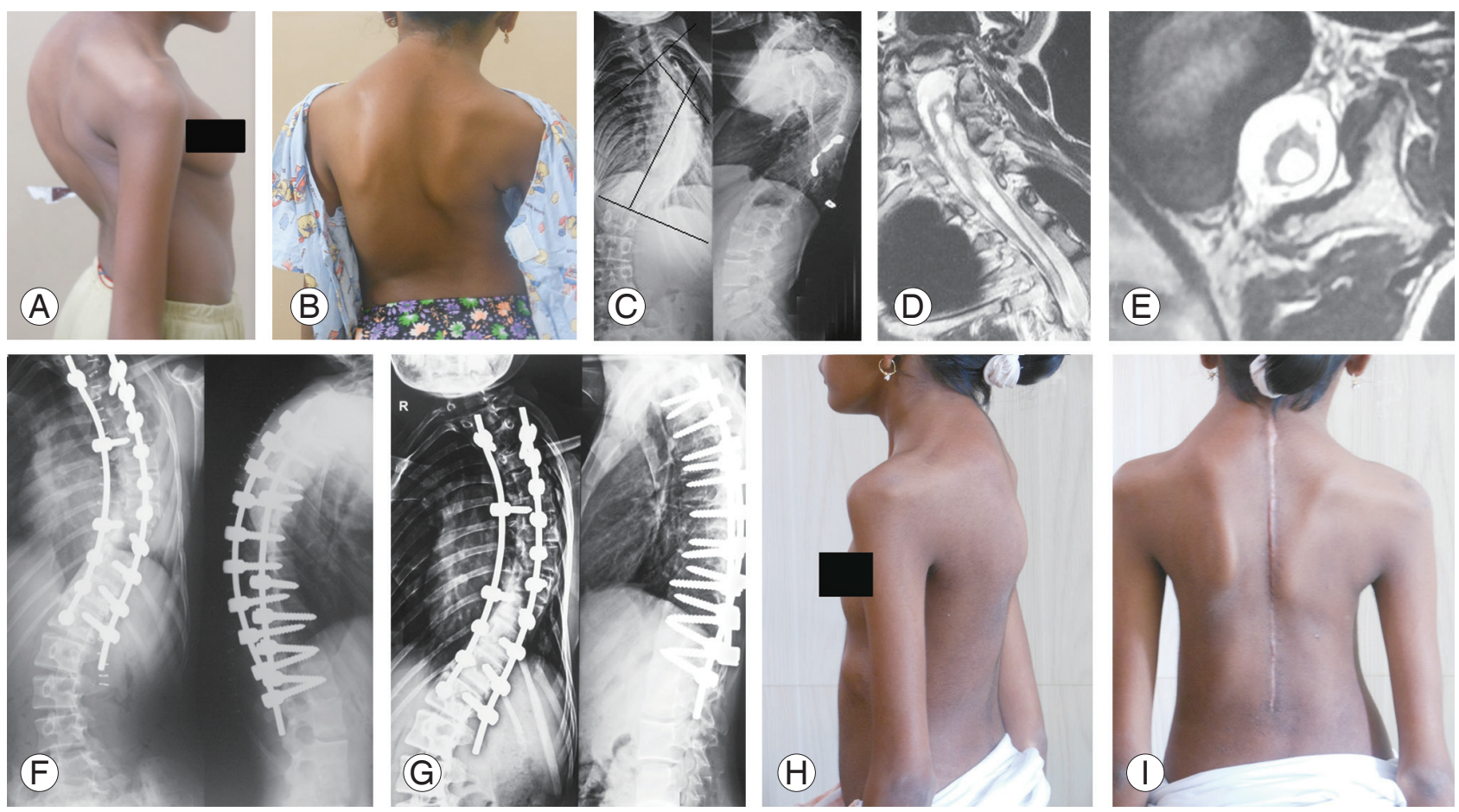

Fig. 2. A 13-year-old girl presented with dorsolumbar kyphoscoliosis with syringomyelia from C5 to D3. She underwent the simultaneous shunting of syringomyelia and posterior instrumentation with pedicle screws and rod from D2 to L1. (A, B) Preoperative lateral and posterior views of the patient showing severe kyphoscoliosis. (C) Preoperative X-ray showing severe kyphoscoliosis with an angle of $63^{\circ}$, with coronal imbalance of 1.1 $\mathrm{cm}$ and sagittal imbalance of $1.4 \mathrm{~cm}$. (D, E) Preoperative MRI with sagittal and axial views showing syringomyelia at the cervicodorsal junction. (F) Postoperative $X$-rays showing deformity correction with postoperative Cobbs angle of $32^{\circ}$, with coronal imbalance of $0.4 \mathrm{~mm}$ and sagittal imbalance of $0.7 \mathrm{~mm}$. (G) Three-year postoperative X-ray showing well-maintained correction. (H, I) Postoperative images of the patient showing good trunk balance.

showed an immediate recovery of power and their neurological status recovered in the immediate postoperative period after extubation. However, in one patient, the neurological status did not show immediate recovery even after the release of the correction but gradually improved and showed a complete recovery after 3 months. None of the patients developed neurological deficits during the surgical treatment of intraspinal anomalies. Thus, the neurological deficits may have occurred due to the overshortening or overstretching of the spinal column during deformity correction. One patient experienced dural tear, which was repaired during the surgery and was uneventfully healed during the postoperative period. Two patients showed pseudoarthrosis with a loss of correction during the follow-up, which required the revision of instrumentation and extension of the fixation cranially and caudally. After revision and extension of the fixation, deformity correction was achieved in both patients.

\section{Discussion}

Congenital spinal deformity associated with intraspinal anomalies leads to rapid curve progression when left untreated $[7,8]$. The incidence of curve progression even after the surgical treatment of intraspinal anomalies was $83 \%$ in a study conducted by Mehta et al. [8]. The chances of curve progression is higher in patients aged $>11$ years, in the presence of neurological symptoms, rotation of vertebral bodies, double scoliosis curve, and curve with an angle of $>50^{\circ}[10]$. With the advent of MRI, the prevalence of intraspinal anomalies has increased to $15 \%-38 \%$ from that reported previously [3]. In our study, tethered cord and diastematomyelia were the most common intraspinal anomalies that presented along with congenital spinal deformities.

Simultaneous surgical intervention for patients with intraspinal anomalies and associated congenital deformity is aimed at preventing deformity progression, treating intraspinal anomalies to prevent neurological deficits, re- 

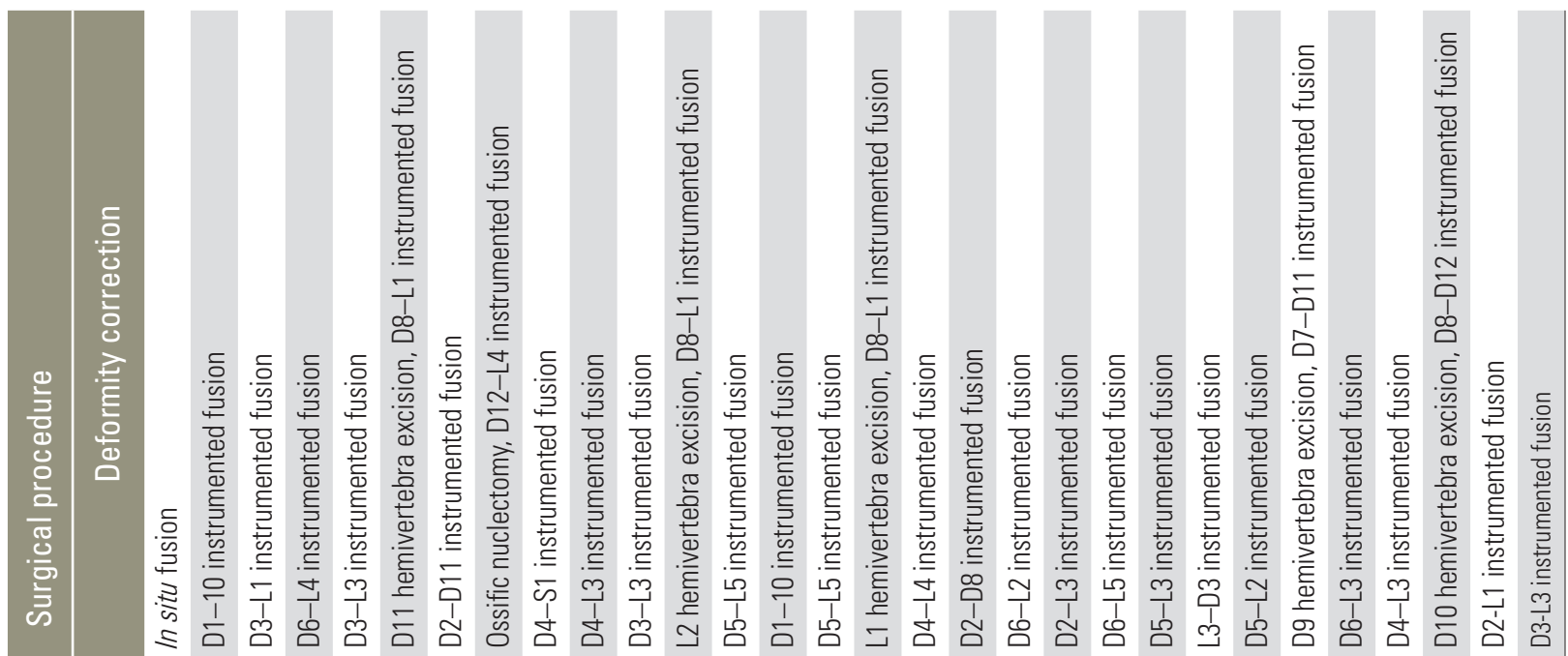

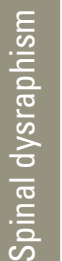

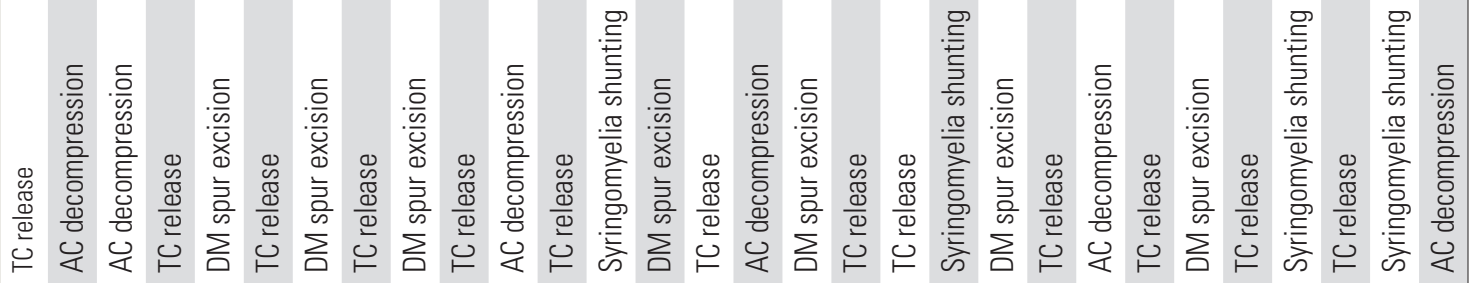

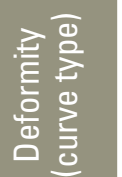

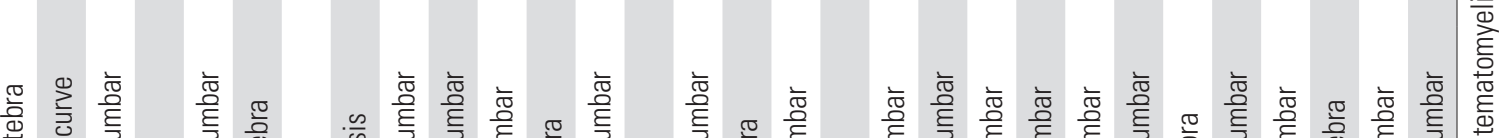

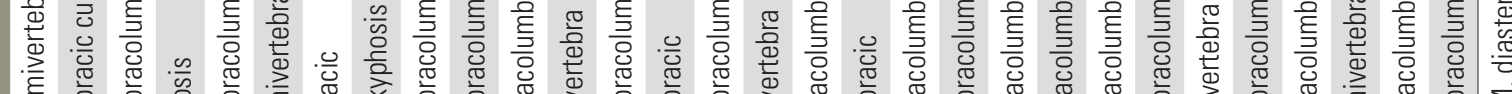

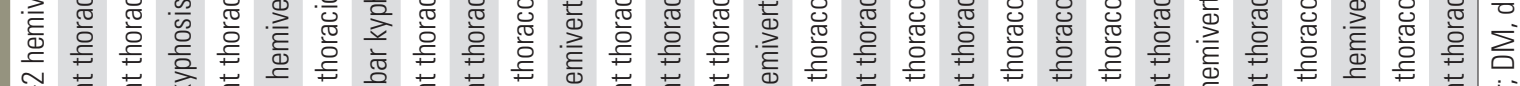

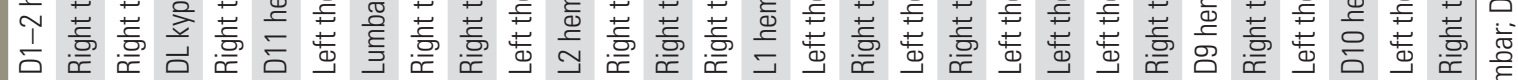

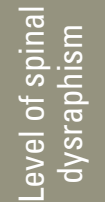

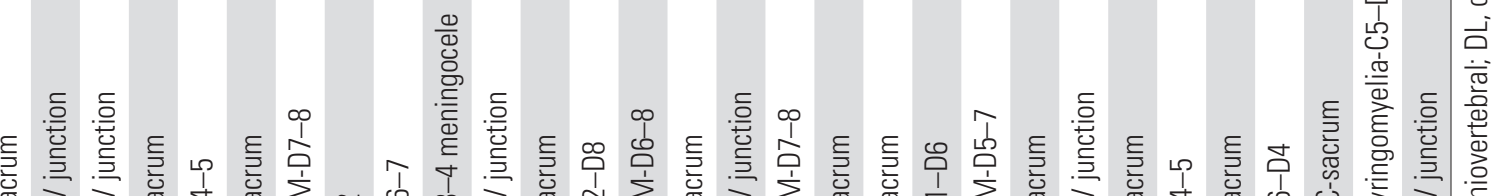

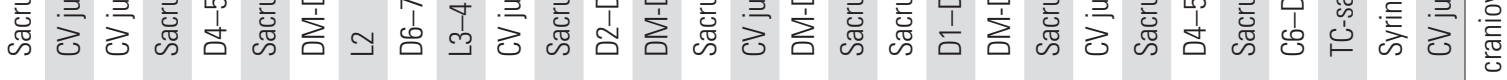

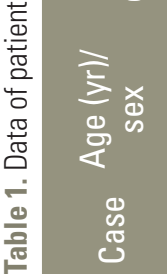

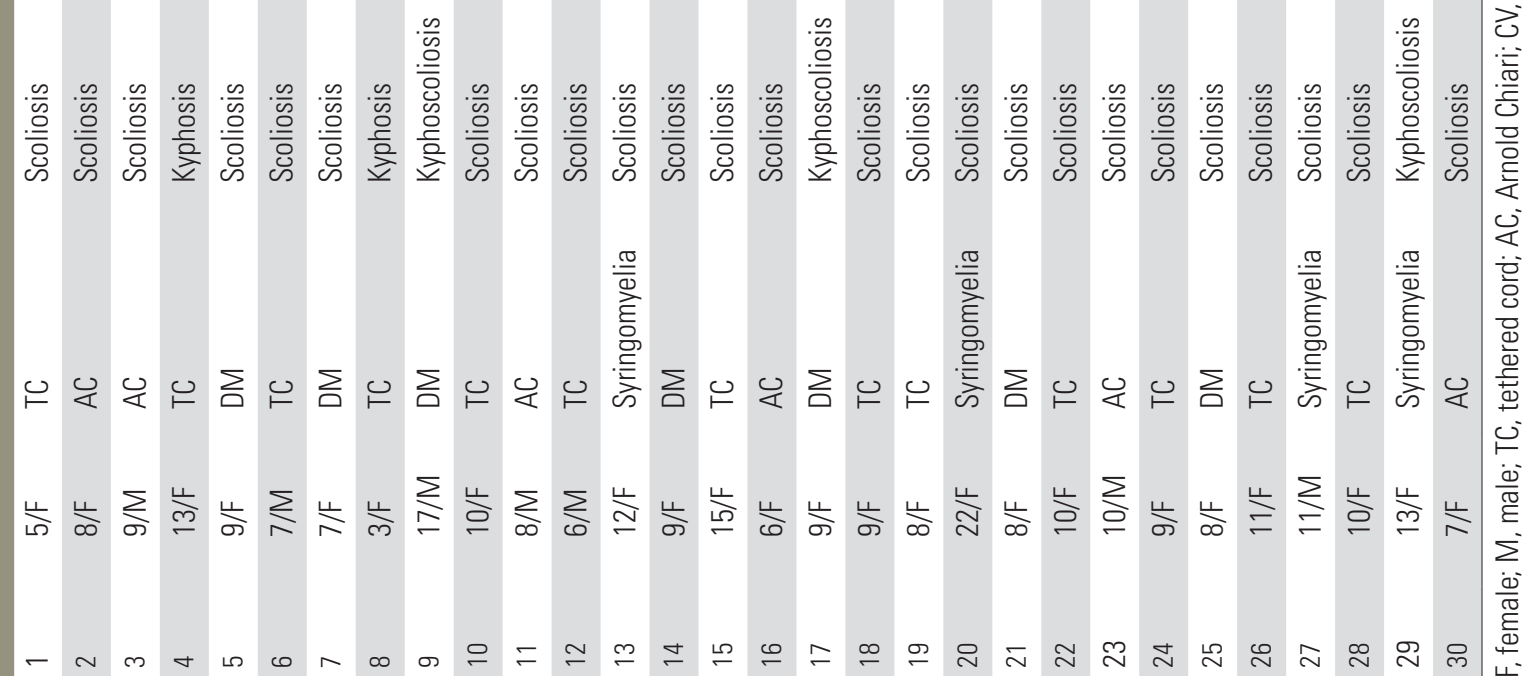




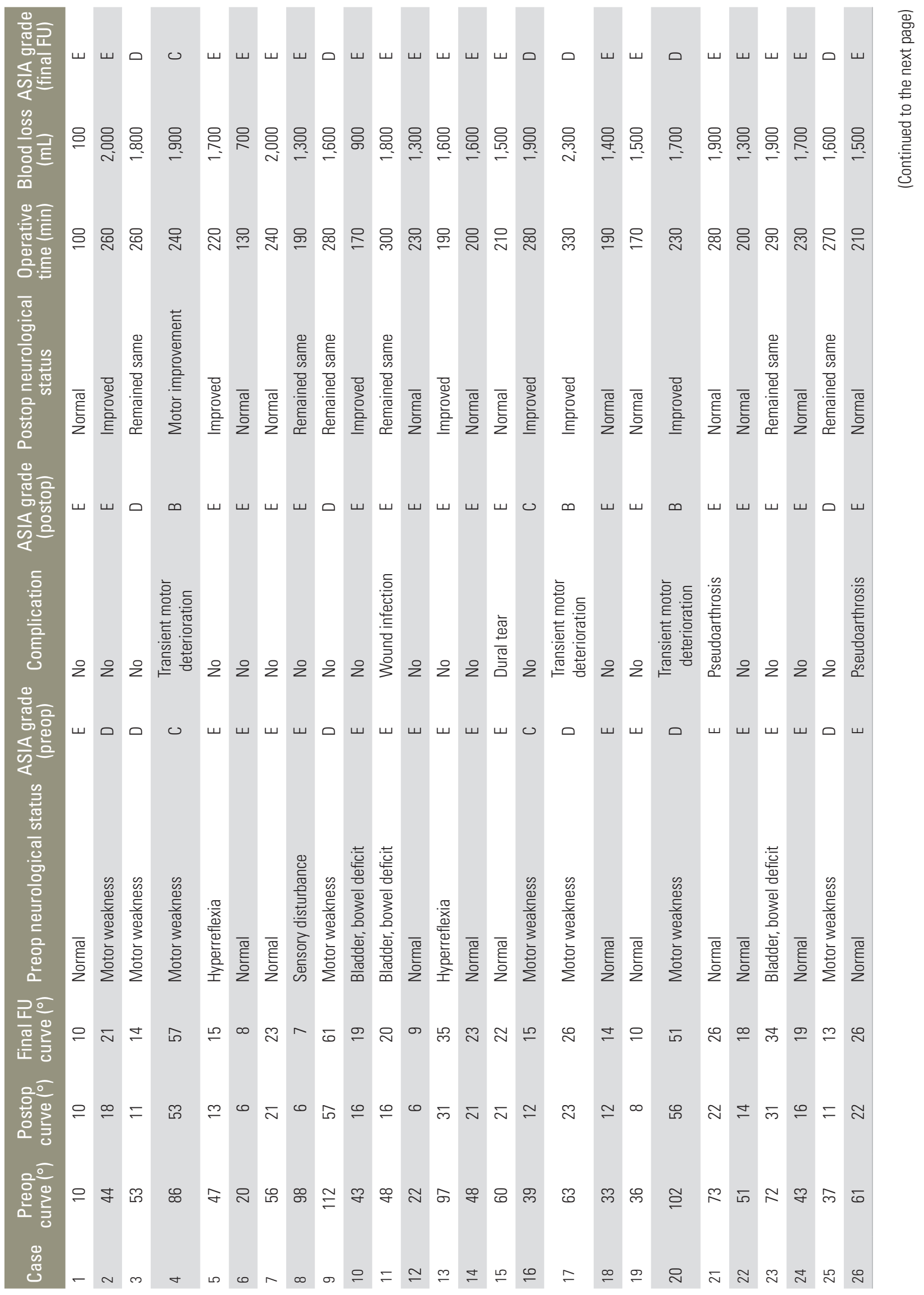




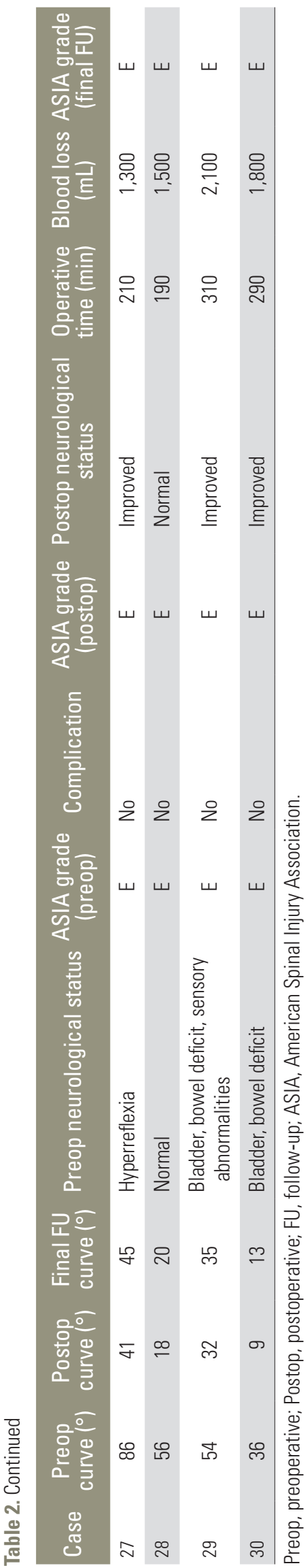

ducing the total duration of treatment and rehabilitation, and minimizing the financial burden on patients' families and the healthcare system [12]. Literature reports on the choice of using single-stage or multi-stage surgery are controversial $[2,4,7,8]$.

In our study, 30 patients were treated using simultaneous surgical intervention for their complex pathologies to reduce the rehabilitation and recuperation period with maximum cost-effectiveness. During the surgery, intraspinal anomalies were treated first, followed by a wake-up test. Following this, deformity correction was performed, which was again followed by a wake-up test. Surgical decisions to perform posterior in situ fusion or corrective deformity surgery was dependent on the age of the patient at presentation, natural history of the congenital anomalous vertebrae, flexibility of the curve, and the preoperative neurological status [13]. One patient in our series required surgery for intraspinal tethered cord with posterior in situ fusion and showed no progress of the deformity at the final follow-up. Localized hemivertebra require short segment fusion with the resection of the hemivertebra $[14,15]$. Long fusion is needed for rigid long-standing deformities requiring corrective osteotomies [16].

In our series, the average age of patients at presentation to the hospital was $9.8 \pm 3.7$ years. Despite the presence of a congenital anomaly at birth, most clinical manifestations of the deformities occur during the second growth spurt when the deformities become much more visible. Neurological deterioration in the patients was also a reason for their presentation to the hospital. In our study, nine patients presented with neurological deficits, three presented with hyperreflexia, and five presented with bladder symptoms (Tables 1, 2). After surgical intervention, neurological deficit worsened in three patients intraoperatively, which was identified during the wake-up test. The neurological status improved in 11 patients compared with the preoperative condition Thus, in our study, neurological status improved in 11 (64.7\%) of 17 patients, compared with the preoperative values, whereas it remained the same as the preoperative values in three (35.2\%). In their study on spinal osteotomy for congenital deformity with diastematomyelia, Chen et al. [2] reported that the neurological status improved in $66.7 \%$ of the patients but not in the remaining $33.3 \%$ of them. Hamzaoglu et al. [7] reported an improvement of the neurological status in $50 \%$ of their patients but no improvement in $25 \%$ of them. Our findings are similar to those of previous studies that 
showed that simultaneous surgical intervention not only prevents neurological deterioration but also improves the neurological status. However, patients and their families should be preoperatively counseled regarding the chances of the improvement of the neurological status.

In their study on congenital scoliosis with intraspinal anomaly, Winter et al. [11] recommended the neurosurgical treatment of the intraspinal anomaly, followed by scoliosis or kyphosis correction, instrumentation, and fusion after a period of 3-6 months. However, such staged surgery is associated with disadvantages such as the risks of two surgeries, revision exposure with more bleeding, loss of normal anatomical landmarks for instrumentation, increased chances of infection, adhesion formation, and re-tethering of the intraspinal anomalies before deformity correction. The risk of iatrogenic neurological trauma increases with revision or second stage surgery $[7,8]$. Mehta et al. [8] compared patients who underwent simultaneous surgery with those who underwent two-stage surgery and showed that the risk of complications such as dural tear, neurological deficit, wound infection, chances of retethering, and pseudoarthrosis rates was significantly higher in patients who underwent the two-stage surgery. In their study on the simultaneous treatment of intraspinal anomalies with deformity correction, Hamzaoglu et al. [7] showed a mean deformity correction of $23 \%$ after an average follow-up duration of 6.8 years; none of the patients had neurological deterioration, pseudoarthrosis, loss of correction, or infection. In their study on 45 patients for the simultaneous treatment of diastematomyelia with deformity correction, Hui et al. [4] showed mean corrections ranging from $73.7^{\circ}$ to $33.5^{\circ}$, with a correction rate of 54.5\%; two patients had transient neurological deterioration $(4.44 \%)$ and one had cerebrospinal fluid (CSF) leak (2.22\%). In their study on single-stage correction for rigid scoliosis with split cord malformation, Chen et al. [2] showed an average correction from $97.2^{\circ}$ to $35.7^{\circ}$, with a correction rate of $64.3 \%$; three patients had transient neurological deterioration (10.3\%), two had CSF leak (6.89\%), one had urinary tract infection (3.44\%), and one had pleural rupture (3.44\%). In our study, the mean deformity correction rate was $62.46 \%$, which is similar to that reported by Hui et al. [4] and Chen et al. [2].

In their study on complications after three-column osteotomy for spinal deformity, Auerbach et al. [17] showed an overall complication rate of $24.8 \%$. A study conducted by Murans et al. [18] on one-stage deformity correction
With or without the treatment of intraspinal anomaly showed an overall complication rate of $34.6 \%$ [18]. A study conducted by Reames et al. [19] on the surgical complications of scoliosis correction in 19,360 patients showed a $10.6 \%$ risk of complications in the surgical treatment. In their study, the incidence of neurological deficit in congenital scoliosis was $2.2 \%$ at long-term follow-up. Reames et al. [19] also showed a similar infection rate in congenital scoliosis at $2.1 \%$. A study conducted by Mehta et al. [8] showed $6.7 \%$ incidence of pseudoarthrosis, which required a revision of implant. In their study on complications of posterior vertebral column resection for spinal deformity, Kim et al. [20] reported an overall complication rate of $40.3 \%$, with neurological deficit in $14.0 \%$ and deformity progression in $10.7 \%$ of the patients.

The complication rate observed in our study $(32.3 \%)$ is consistent with that reported in previous studies. Transient neurological deficit was observed in three patients (9.6\%), of whom two immediately regained power on the release of correction and one gradually regained power over 3 months. Two patients (5.8\%) had implant failure due to pseudoarthrosis, which led to deformity progression; both these patients underwent revision surgery with the removal of loose implants, re-fixation with pedicle screws with the extension of construct proximally and distally, along with iliac crest bone grafting. After the revision surgery, both patients showed good deformity correction without any neurological deficit. Wound infection and CSF leak occurred in one patient (3.33\%).

There were certain limitations to our study. First, there was no control group in which multiple-stage surgery was performed to compare the outcomes and complication rates. Second, our study included a small group of patients because the incidence of disease is rare. However, the outcomes and complication rates reported in our study were comparable to those reported in similar previous studies.

The findings of transient neurological deficit with the complete recovery of motor power, pseudoarthrosis, and infection rates in our study were comparable to those of previous studies. The similar complication rates observed in our study and in previous studies on the single-stage treatment of spinal deformity and intraspinal anomalies, along with good postoperative deformity correction rates, support our hypothesis that a single-stage surgery is preferable to multiple-stage surgery in avoiding the risks and complications associated with revision surgery, reducing the financial burden on the family, and decreasing the du- 
ration of rehabilitation and recuperation.

\section{Conclusions}

Simultaneous surgical treatment of intraspinal anomalies and congenital spinal deformity correction, including adequate intra-operative wake-up test, is a viable option in appropriately selected patients and has minimal complication rates. Simultaneous surgical intervention helps to reduce the duration of rehabilitation and recuperation and avoids the need for repeated hospitalizations and the risks of repeated anesthesia.

\section{Conflict of Interest}

No potential conflict of interest relevant to this article was reported.

\section{References}

1. Gupta N, S R, G B, Shetty A. Vertebral and intraspinal anomalies in Indian population with congenital scoliosis: a study of 119 consecutive patients. Asian Spine J 2016;10:276-81.

2. Chen B, Yuan Z, Chang MS, et al. Safety and efficacy of one-stage spinal osteotomy for severe and rigid congenital scoliosis associated with split spinal cord malformation. Spine (Phila Pa 1976) 2015;40:E100513.

3. Shen J, Wang Z, Liu J, Xue X, Qiu G. Abnormalities associated with congenital scoliosis: a retrospective study of 226 Chinese surgical cases. Spine (Phila Pa 1976) 2013;38:814-8.

4. Hui H, Tao HR, Jiang XF, Fan HB, Yan M, Luo ZJ. Safety and efficacy of 1-stage surgical treatment of congenital spinal deformity associated with split spinal cord malformation. Spine (Phila $\mathrm{Pa} 1976$ ) 2012;37:2104-13.

5. Kaplan KM, Spivak JM, Bendo JA. Embryology of the spine and associated congenital abnormalities. Spine J 2005;5:564-76.

6. Belmont PJ Jr, Kuklo TR, Taylor KF, Freedman BA, Prahinski JR, Kruse RW. Intraspinal anomalies associated with isolated congenital hemivertebra: the role of routine magnetic resonance imaging. J Bone Joint Surg Am 2004;86-A:1704-10.

7. Hamzaoglu A, Ozturk C, Tezer M, Aydogan M,
Sarier M, Talu U. Simultaneous surgical treatment in congenital scoliosis and/or kyphosis associated with intraspinal abnormalities. Spine (Phila Pa 1976) 2007;32:2880-4.

8. Mehta VA, Gottfried ON, McGirt MJ, Gokaslan ZL, Ahn ES, Jallo GI. Safety and efficacy of concurrent pediatric spinal cord untethering and deformity correction. J Spinal Disord Tech 2011;24:401-5.

9. Rajasekaran S, Kamath V, Kiran R, Shetty AP. Intraspinal anomalies in scoliosis: an MRI analysis of 177 consecutive scoliosis patients. Indian J Orthop 2010;44:57-63.

10. Ameri E, Andalib A, Tari HV, Ghandhari H. The role of routine preoperative magnetic resonance imaging in idiopathic scoliosis: a ten years review. Asian Spine J 2015;9:511-6.

11. Winter RB, Haven JJ, Moe JH, Lagaard SM. Diastematomyelia and congenital spine deformities. J Bone Joint Surg Am 1974;56:27-39.

12. Samdani AF, Asghar J, Pahys J, D’Andrea L, Betz RR. Concurrent spinal cord untethering and scoliosis correction: case report. Spine (Phila Pa 1976) 2007;32:E832-6.

13. Smith JS, Abel MF, Shaffrey CI, Ames CP. Decision making in pediatric spinal deformity. Neurosurgery 2008;63(3 Suppl):54-68.

14. Shono Y, Abumi K, Kaneda K. One-stage posterior hemivertebra resection and correction using segmental posterior instrumentation. Spine (Phila Pa 1976) 2001;26:752-7.

15. Zhang J, Shengru W, Qiu G, Yu B, Yipeng W, Luk KD. The efficacy and complications of posterior hemivertebra resection. Eur Spine J 2011;20:1692-702.

16. Gill JB, Levin A, Burd T, Longley M. Corrective osteotomies in spine surgery. J Bone Joint Surg Am 2008;90:2509-20.

17. Auerbach JD, Lenke LG, Bridwell KH, et al. Major complications and comparison between 3-column osteotomy techniques in 105 consecutive spinal deformity procedures. Spine (Phila Pa 1976) 2012;37:1198-210.

18. Murans G, Gustavsson B, Saraste H. One-stage major spine deformity correction surgery: comparison between groups with and without additional neurosurgical intervention, with more than 24 months of follow-up: clinical article. J Neurosurg Spine 2010;13:666-71. 
19. Reames DL, Smith JS, Fu KM, et al. Complications in the surgical treatment of 19,360 cases of pediatric scoliosis: a review of the Scoliosis Research Society Morbidity and Mortality database. Spine (Phila Pa 1976) 2011;36:1484-91.
20. Kim SS, Cho BC, Kim JH, et al. Complications of posterior vertebral resection for spinal deformity. Asian Spine J 2012;6:257-65. 